\title{
Changes in Bone Mineral Density Following Weight Loss Induced by One-Anastomosis Gastric Bypass in Patients with Vitamin D Supplementation
}

\author{
Maria Luger ${ }^{1,2,3} \cdot$ Renate Kruschitz $^{1,4} \cdot$ Eva Winzer $^{2,3}$ (D) Karin Schindler ${ }^{1} \cdot$ Igor Grabovac ${ }^{3}$ - Franz Kainberger ${ }^{5}$. \\ Michael Krebs ${ }^{1} \cdot$ Friedrich Hoppichler $^{2,6} \cdot$ Felix Langer $^{7} \cdot$ Gerhard Prager $^{7} \cdot$ Rodrig Marculescu $^{8}$ • Bernhard Ludvik ${ }^{1,9}$
}

Published online: 2 July 2018

(C) The Author(s) 2018

\begin{abstract}
Background Little is known about changes in bone mineral density (BMD) following weight loss after one-anastomosis gastric bypass (OAGB) and the role of serum vitamin $\mathrm{D}$ and its supplementation on bone metabolism. We evaluated BMD after OAGB as a function of vitamin D supplementation with respect to a minimum threshold of 25-hydroxy-vitamin-D [25(OH)D] concentration, which could prevent or decelerate an eventual bone loss.

Methods Fifty bariatric patients who participated in the randomized controlled trial were included in this analysis. BMD and anthropometric measurements by DXA and laboratory parameters were assessed before (T0), at 6 (T6), and 12 months (T12) after surgery.

Results OAGB resulted in a $36 \%$ total body weight loss with a decrease in body fat and an increase in lean body mass. A significant decrease in BMD was seen in lumbar spine by $7 \%$, left hip 13\%, and total body $1 \%$, but not in forearm. Bone turnover markers increased significantly but with normal parathyroid hormone concentrations. Weight loss was not associated with changes in BMD. A serum 25(OH)D concentration $>50 \mathrm{nmol} / 1$ at T6 and T12 (adequate-vitamin-D-group; AVD) showed a significant lower bone loss, compared to the inadequate-vitamin-D-group (IVD; $<50 \mathrm{nmol} / \mathrm{l}$ ). Lower bone loss in the left hip showed a strong correlation with higher $25(\mathrm{OH}) \mathrm{D}$ concentrations $(r=0.635, p=0.003)$.

Conclusion These findings support a dose effect of vitamin D supplementation on bone health and suggest that 25(OH)D concentrations need to be above $50 \mathrm{nmol} / 1$ at least during the first postoperative year to decelerate bone loss in patients undergoing OAGB.

Clinical Trial Registry Number and Website Clinicaltrials.gov (NCT02092376) at https://clinicaltrials.gov/.

EudraCT (2013-003546-16) at https://eudract.ema.europa.eu/.
\end{abstract}

Keywords Bone loss $\cdot$ Vitamin D $\cdot$ Obesity $\cdot$ One-anastomosis gastric bypass $\cdot$ Weight loss

Eva Winzer

eva.winzer@meduniwien.ac.at

Maria Luger

maria.luger@medunwien.ac.at

Renate Kruschitz

renate.kruschitz@meduniwien.ac.at

Karin Schindler

karin.schindler@meduniwien.ac.at

Igor Grabovac

igor.grabovac@meduniwien.ac.at

Franz Kainberger

franz.kainberger@meduniwien.ac.at

\author{
Michael Krebs \\ michael.krebs@meduniwien.ac.at \\ Friedrich Hoppichler \\ office@sipcan.at \\ Felix Langer \\ felix.langer@meduniwien.ac.at \\ Gerhard Prager \\ gerhard.prager@meduniwien.ac.at \\ Rodrig Marculescu \\ rodrig.marculescu@meduniwien.ac.at \\ Bernhard Ludvik \\ bernhard.ludvik@wienkav.at
}

Extended author information available on the last page of the article 


\section{Introduction}

Little is known about changes in bone mineral density (BMD) following weight loss after one-anastomosis gastric bypass (OAGB). Bariatric surgery is an effective method to treat morbid obesity, its comorbidities such as (pre)diabetes $[1,2]$ and non-alcoholic fatty liver disease (NAFLD) [3], and is associated with long-term weight loss and decreased overall mortality [4]. Due to gastric restriction and malabsorption by the gastric bypass procedure, $50-96 \%$ of the patients exhibit nutrition-related deficiencies such as vitamin D deficiency [5-8]. Thus, low vitamin D concentrations and elevated parathyroid hormone (PTH) levels as mediators of bone loss are common findings in obese patients. After bariatric surgery, vitamin $\mathrm{D}$ deficiency and secondary hyperparathyroidism may be exacerbated by the malabsorptive state.

Bariatric surgery also affects bone metabolism by decreasing mechanical loading and subsequent reduction in the differentiation of osteoblasts [9]. In addition, loss of adipose tissue mass and changes in anatomy and physiology of the gastrointestinal tract lead to altered hormone and adipokine secretion further affecting bone metabolism [9].

The association between vitamin D deficiency and bone disease is well described [10], and malabsorption following bariatric surgery is regarded the main cause for postoperative bone loss [11]. OAGB is a quite recently developed bariatric procedure with higher weight loss compared to the traditional Roux-en-Y gastric bypass (RYGB). Both procedures have restrictive as well as malabsorptive properties [12]. Additionally, there is an increasing body of literature suggesting a role for appetite suppression mediated by neurohormonal signals after RYGB [13]. Regarding the best approach for treating vitamin D deficiency in bariatric patients, only limited data are available, especially for OAGB. In postmenopausal women, vitamin D supplementation slows bone loss [14], protects against weakness and the risk of falling [15], and decreases secondary hyperparathyroidism [16]. In bariatric patients, even with a very high vitamin D dose [17], it has been challenging to achieve the recommended 25hydroxy-vitamin D [25(OH)D] concentrations above $75 \mathrm{nmol} / 1$ [8].

Obesity has been regarded as protective against osteoporosis because body weight amplifies bone density [18]. After RYGB, the rate of bone loss is greatest during the first year and plateaus or even slightly improves in subsequent years [19]. This is considered to be the consequence of surgically induced weight loss. In that regard, postoperative bone loss seems to be a dynamic process and the role of vitamin D deficiency is not fully elucidated nor has the use of supplements been proven to reverse this process [20]. Vitamin D status might be a modifying factor in bone loss but the requirements of vitamin D supplementation or a $25(\mathrm{OH}) \mathrm{D}$ concentration which is adequate after OAGB remains elusive and to our knowledge has not been studied in those patients. Additionally, as the prevalence of bariatric surgeries increases, there is a need for more information about its potential deleterious effects on bones $[9,11]$.

As part of a randomized controlled trial of vitamin $D_{3}$ supplementation after OAGB, safety and efficacy of high-dose vitamin D supplementation were previously reported [21]; however, changes of BMD have not been investigated so far. As achieving sufficient $25(\mathrm{OH}) \mathrm{D}$ concentrations postoperatively has been challenging, it was hypothesized that highdose oral vitamin $\mathrm{D}_{3}$ supplementation leading higher $25(\mathrm{OH}) \mathrm{D}$ concentrations would result in a deceleration or prevention of bone loss. Therefore, the aim of this analysis was to determine the effects of vitamin D levels on BMD and parameters of bone turnover following OAGB-induced weight loss.

\section{Methods}

\section{Study Design}

This study was approved by the local Ethics Committee of the Medical University of Vienna (No. 1899/2013), by the Austrian Competent Authority (No. LCM-718280-0001), registered at clinicaltrials.gov (Identifier: NCT02092376) and EudraCT (Identifier: 2013-003546-16), complies with the Declaration of Helsinki [22], and conducted from April 2014 to June 2016 at the Medical University of Vienna (Austria). The study participants were scheduled for OAGB surgery and all of them gave written, informed consent preoperatively. Subjects were participants in a 6-month double-blind, placebo-controlled, randomized trial of vitamin D supplementation, the LOAD-study ("Link between Obesity And Vitamin D") [21]. The study protocol was previously published [23]. Participants were randomly assigned to one of two vitamin D supplementation groups. The intervention group received three oral vitamin $\mathrm{D}_{3}$ loading doses of each $100,000 \mathrm{IU}$ in the first month postoperatively followed by maintenance dose of $3420 \mathrm{IU} /$ day until 6 months with compliance testing. The control group obtained placebo in the first month followed by maintenance dose of 3420 IU/day until 6 months. Afterwards, both groups were recommended to continue the vitamin $\mathrm{D}_{3}$ supplementation until the follow-up visit at 12 months. Supplementation adherence was reviewed by medication counts until the end of the trial 6 months after surgery (T6). At 12 months postoperatively (T12), the adherence was not assessed. Regarding the first, second, and third loading doses in the first month postoperatively, 100, 100, and $96 \%$ of the participants took their assigned study drug (cholecalciferol) or placebo. Adherence to the subsequent maintenance dose was at 2,3,4,5, and 6 months after surgery at $67,70,71,63$, and $61 \%$, respectively, without statistically significant differences between the study groups [21]. The 
reason given by the patients was that they "have forgotten to take the vitamin D supplementation every week", despite a reminder on a regular basis. At baseline, no participant took any vitamin $\mathrm{D}$ supplementation, which was an inclusion criterion for this trial. Inclusion criteria were that the patients were planned for OAGB surgery, were above 18 years old, had a serum $25(\mathrm{OH}) \mathrm{D}$ concentrations of $<75 \mathrm{nmol} / \mathrm{l}$, and a body weight $<140 \mathrm{~kg}$ (due to body weight limitation of the dual-energy X-ray absorptiometry). Specific exclusion criteria included any other planned form of bariatric surgery than OAGB, hypo- and hypercalcemia, renal insufficiency, or primary hyperparathyroidism. The details on design, the used materials and methods, as well as the sample size calculation of the study have been previously published [23]. The findings regarding safety and efficacy $[25(\mathrm{OH}) \mathrm{D}$ as main outcome parameter] at $\mathrm{T} 6$ of the trial have been previously published [21]. Compared with control group, a higher increase of 25(OH)D concentration $(67.9$ (SD: 21.1$)$ vs. $55.7 \mathrm{nmol} / 1$ $(21.1) ; p=0.049)$ with lower prevalence of secondary hyperparathyroidism (10 vs. $24 \% ; p=0.045)$ was observed in the intervention group [21].

All OAGB procedures were performed at the General Hospital Vienna, Medical University of Vienna by the same surgical team using a laparoscopic approach. It is a simplified procedure that consists of a unique gastrojejunal anastomosis between a 30- and 40-ml sleeve gastric pouch and a jejunal omega-loop of approximately $200 \mathrm{~cm}$ [24]. The study methods are in accordance with the CONSORT (Consolidated Standards Of Reporting Trials) guidelines for reporting randomized trials [25].

\section{Vitamin D Groups}

The 50 bariatric patients represent the participants of the randomized controlled trial (Fig. 1). Out of 67 eligible patients, $25 \%$ declined to participate, and 50 patients were included at baseline [21]. After randomization, four randomization failures occurred and were excluded from the study. In total, the drop-out rate was $6.5 \%(n=3)$ at 6 months $(\mathrm{T} 6)$ and $19.6 \%$ $(n=9)$ at 12 months postoperatively (T12).

For the purposes of this analysis, vitamin $\mathrm{D}$ inadequacy was defined as a $25(\mathrm{OH}) \mathrm{D}$ concentration $<50 \mathrm{nmol} / \mathrm{l}(<$ $20 \mathrm{ng} / \mathrm{ml}$ ), and vitamin D adequacy was defined as a $25(\mathrm{OH}) \mathrm{D}$ concentration $\geq 50 \mathrm{nmol} / 1(\geq 20 \mathrm{ng} / \mathrm{ml})$. Accordingly and based on calculations, the study population was divided in patients who demonstrated $25(\mathrm{OH}) \mathrm{D}$ concentrations $\geq 50 \mathrm{nmol} / 1$ at $\mathrm{T} 6$ and at $\mathrm{T} 12$ (adequate vitamin $\mathrm{D}$ group; AVD) and in those $<50 \mathrm{nmol} / 1$ at $\mathrm{T} 6$ and/ or T12 (inadequate vitamin D group; IVD). This cut-off of $25(\mathrm{OH}) \mathrm{D}$ concentrations was assessed due to following considerations and calculations: to examine the cut-off value of $25(\mathrm{OH}) \mathrm{D}$ in the first year in patients undergoing OAGB to prevent or decelerate bone loss, we used the receiver operating characteristic (ROC) curve of $25(\mathrm{OH}) \mathrm{D}$ and the t-scores of all four regions. T-scores represent numbers that compare the condition of the bones with those of an average young person with healthy bones and are usually in the negative or minus range. The World Health Organization (WHO) in 1994 gave operational definition of osteoporosis as follows: normal bone density: T-score between +1 and -1 ; osteopenia or low bone mass: T-score between -1.1 and -2.4 ; osteoporosis: T-score of -2.5 or less [26]. By using t-score of lumbar spine below 2.5 (osteoporosis), the ROC curve demonstrated an area under the curve (AUC) of $0.682(0.15)$. A $25(\mathrm{OH}) \mathrm{D}$ concentration $\geq$ $50 \mathrm{nmol} / 1$ had a sensitivity of $50 \%$ and a specificity of $77 \%$ and a cut-off of $\geq 75 \mathrm{nmol} / \mathrm{l}$ had a sensitivity of $100 \%$ but lower specificity of $32 \%$. We used the T-score of the lumbar spine, as only in this region we could observe t-scores below -2.5 and the ROC calculation showed the highest AUC compared to other BMD regions. Therefore, for further analyses and for the vitamin D groups, we used the cut-off value of $50 \mathrm{nmol} / \mathrm{l}$.

\section{Assessment of Variables}

Data were assessed before study begin (T0), at 6 months (T6), and at follow-up visit at 12 months (T12). At T0, age, sex, and medical history (e.g., comorbidities, prescribed medication) were collected as previously described [23]. The following set of evaluations was obtained for each participant at the three time points: height and body weight (measured with the calibrated scale seca mBCA 515) and waist circumference measured with an inelastic tape at the narrowest point between the lower costal border and the top of the iliac crest in accordance with the International Standards for Anthropometric Assessment (ISAK) [27].

Blood samples were collected, and following laboratory parameters were used for this analysis: albumin $(\mathrm{g} / \mathrm{dl})$, serum type 1 collagen cross-linked C-telopeptide (CTX; ng/ml), osteocalcin (ng/ml), bone-specific alkaline phosphatase (ng/ $\mathrm{ml}$ ), intact amino terminal propeptide of type 1 procollagen (P1NP; mg/l), intact parathyroid hormone iPTH $(\mathrm{pg} / \mathrm{ml})$, $25(\mathrm{OH}) \mathrm{D}(\mathrm{nmol} / \mathrm{l}), 1,25(\mathrm{OH})_{2} \mathrm{D}(\mathrm{pg} / \mathrm{ml})$, albumin-corrected calcium $(\mathrm{Ca} ; \mathrm{mmol} / \mathrm{l})[28])$, and phosphate $(\mathrm{mmol} / \mathrm{l})$. A chemiluminescence immunoassay was used to measure the total circulating $25(\mathrm{OH}) \mathrm{D}$ concentration in serum samples. This immunoassay is an accredited test procedure and the laboratory participated in the study from the very beginning to ensure reliability of the $25(\mathrm{OH}) \mathrm{D}$ assays.

BMD (grams per centimeter ${ }^{2}$ ) and bone mineral content (BMC; grams) were assessed at the posteroanterior lumbar spine (L1-L4), left hip (femoral neck, trochanter, and total hip), forearm (one-third distal radius, ultradistal, and total radius), and total body by dual-energy x-ray absorptiometry (DXA, Hologic Discovery A; S/N 45312, Hologic, Inc., Bedford, MA) and were acquired according to the procedures 


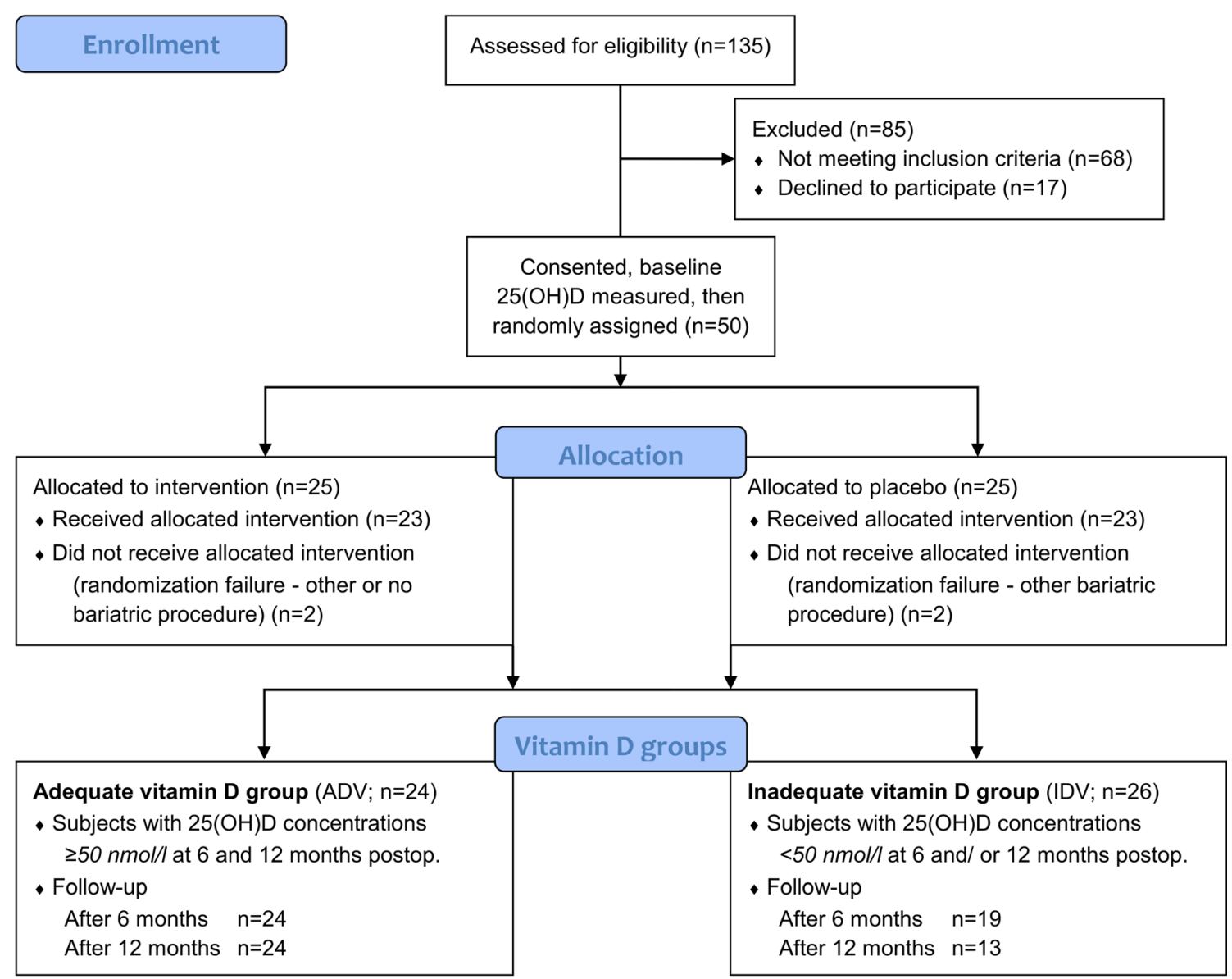

Fig. 1 CONSORT flow chart of participants in the randomized controlled trial and the used vitamin D groups

recommended by the manufacturer and by the guidelines of the International Society of Clinical Densitometry (ISCD) including the International Osteoporosis Foundation-certificate. All DXA scans were done by the same technologist team. Machine calibration and subject positioning during DXA scan were standardized. Body composition was assessed by the whole body fan beam as total body fat (\%) and lean body mass $(\%)$.

\section{Statistical Analysis}

The results are expressed as mean (standard deviation or standard error) for continuous and as percentages for categorical variables. In order to test for normal distribution, a visual test (histograms and box plots) was used and the KolmogorovSmirnov test was applied in addition. Statistical significance tests such as $t$ test or Mann-Whitney $U$ test and $\mathrm{Chi}^{2}$ test were applied to assess differences between the AVD and the IVD group at baseline. The cut-off value for $25(\mathrm{OH}) \mathrm{D}$ in regard to the t-score was assessed using the ROC curve described as AUC with standard errors. The main outcome of interest was the change in bone measures in the first postoperative year. We applied repeated-measures analysis of covariance
(ANCOVA) using random error (linear mixed model) to assess the effect of time, group, and their interaction for changes in parameters between the groups, by using different covariance structure models as appropriate and were adjusted for age, sex, season, and baseline values to supply an unbiased estimate of the mean group difference [29]. Moreover, a post hoc analysis with Bonferroni correction was used. Additionally, linear regression was performed to assess associations between changes in BMD variables to change of variables in body composition, bone turnover, and vitamin D metabolism, adjusted for age, sex, and season. Estimates of the prevalence of osteopenia or -porosis ( $t$-score below -1 or below -2.5 ) between the groups over time were calculated using generalized estimating equation (GEE) with a logit link function for binary outcomes and unstructured covariance matrices. With this approach, we examined effects with time as repeated factor and group as between subject factor with prevalence of osteopenia/-porosis (yes, no) as dependent variable, adjusted for age, sex, season, and baseline values. All statistical analyses were performed with IBM® SPSS ${ }^{\circledR}$ Statistics for Windows, Version 23 software (IBM Corporation, Armonk, New York, USA). $P$ values $<0.05$ were considered statistically significant and all tests were two-sided. 


\section{Results}

\section{Bone Mineral Density, Parameters of Bone Turnover, and Body Composition}

Changes in BMD, parameters of bone turnover, albumin, iPTH, 25(OH)D, 1,25(OH $)_{2} \mathrm{D}$, calcium, phosphate, and body composition are shown in Table 1. All parameters changed significantly at T6, except for iPTH, calcium, BMD lumbar spine (absolute and t-sore), BMD left hip (absolute), BMD forearm (absolute and t-score), and BMD total body (absolute and $\mathrm{t}$-score). The different regions of BMD did not change significantly at $\mathrm{T} 6$, except for left hip t-score. In addition, all parameters changed significantly over the whole time period from baseline until T12, except for iPTH, calcium, and BMD forearm. Over 12 months, a significant decrease in BMD was seen (Fig. 2) when measured in the lumbar spine (7.1\% (standard error: $1.5 \%), p<0.001)$, left hip (12.6\% (1.1\%), $p<$ $0.001)$, and in the total body (1.3\% (0.6), $p=0.046)$, except for forearm $(0.9 \%(0.7 \%), p=0.172)$.

\section{Associations Between Vitamin D and Parameters of Bone Turnover and Laboratory Parameters}

Table 2 shows the comparison between the AVD and the IDV group on parameters of bone turnover and laboratory parameters. At T0, the AVD group showed significantly higher CTX and bone-specific alkaline phosphatase and lower calcium concentrations. At T12, significant group differences in bone-specific alkaline phosphatase and, as expected, in $25(\mathrm{OH}) \mathrm{D}$ could be found. The AVD group had a significantly higher 25(OH)D concentration at T6 and at T12. In addition, calcium showed a significant group and time interaction with higher concentrations in the AVD group.

The significant changes in BMD in absolute values and tscore are shown in Fig. 3 and Fig. 4. The AVD group showed significantly higher BMD values compared to the IDV group, adjusted for age, sex, season, and baseline values. Significant differences in absolute values $\left(\mathrm{g} / \mathrm{cm}^{2}\right)$ could be found between the groups in forearm ( $p=0.043$; Fig. 3c), over time in lumbar spine ( $p<0.001$; Fig. 3a), left hip ( $p<0.001$; Fig. 3b), and total body ( $p=0.006$; Fig. $3 \mathrm{~d}$ ) and a significant interaction between group and time in lumbar spine $(p=0.024)$ and forearm $(p=0.028)$. After 1 year, the relative declines in BMD were lower in the AVD group compared to the IDV group (lumbar spine: 5.7 vs. $10.0 \%$; left hip: 10.0 vs. $17.4 \%$; forearm: 0.5 vs. $1.5 \%$; total body: 0.6 vs. $2.3 \%$ ). Regarding the tscore, significant differences were observed between the groups in left hip ( $p<0.001$; Fig. $4 \mathrm{~b})$ and forearm $(p<$ 0.001 ; Fig. $4 \mathrm{c}$ ), over time in lumbar spine ( $p=0.001$; Fig. $4 a)$, left hip $(p<0.001)$, forearm $(p<0.001)$, and total body $(p=0.001$; Fig. $4 \mathrm{~d})$ and a significant interaction between group and time in lumbar spine $(p=0.029)$, left hip $(p<$
$0.001)$, forearm $(p=0.006)$, and total body $(p=0.026)$. Accordingly, at T12, the absolute declines in t-score were lower in the AVD group compared to the IDV group (lumbar spine: 0.57 vs. 1.06 ; left hip: 0.83 vs. 1.64 ; forearm: 0.08 vs. 0.57 ; total body: 0.07 vs. 0.40 ).

By using generalized estimating equation, adjusted for age, sex, season, and baseline values, the estimates of the prevalence of osteopenia ( $t$-score below - 1) in lumbar spine, left hip, forearm, and total body are presented in Table 3. Regarding the prevalence of osteoporosis ( $\mathrm{t}$-score below $2.5)$ in the lumbar spine, we did not observe a significant difference between the groups $(p=0.405)$ nor over time $(p=$ 0.187; T0: 0 vs. $1 \%$; T6: 1 vs. $1 \%$; T12: 2 vs. $4 \%$ ). In addition, in the other three regions (left hip, forearm, and total body), no patient demonstrated a t-score below -2.5 over time.

No significant associations between the change of body weight or BMI to the change of BMD in all four regions, adjusted for age, sex, and season, were seen in our study. This bone loss appeared independent from weight loss.

Regarding changes in parameters of bone turnover and vitamin D metabolism, we could observe significant associations between the change in total body BMD $\left(\mathrm{g} / \mathrm{m}^{2}\right)$ and the change in CTX $(r=-0.474, p=0.050)$, osteocalcin $(r=-$ $0.572, p=0.009)$, and P1NP $(r=-0.657, p=0.009)$, adjusted for age, sex, and season. Moreover, we assessed significant associations between the change in left hip BMD $\left(\mathrm{g} / \mathrm{m}^{2}\right)$ and the change in calcium $(r=-0.487, p=0.013)$ and change in $25(\mathrm{OH}) \mathrm{D}(r=0.598, p=0.009)$. Additionally, the 25(OH)Dconcentration profile of each bariatric patient was summarized by averaging her/his $25(\mathrm{OH}) \mathrm{D}$ concentrations across visits until T12 and is referred to as mean $25(\mathrm{OH}) \mathrm{D}$. In that regard, the mean $25(\mathrm{OH}) \mathrm{D}$ concentration during surgically induced weight loss was significantly associated with changes in left hip BMD $\left(\mathrm{g} / \mathrm{cm}^{2} ; r=0.671, p=0.002\right.$; adjusted for age, sex, and season).

\section{Discussion}

To our knowledge, this is the first randomized controlled trial that examined BMD during weight loss after one-anastomosis gastric bypass as a function of postoperative vitamin D status. Patients with higher vitamin D levels (serum 25(OH)D concentration above $50 \mathrm{nmol} / \mathrm{l}$ ) in the first postoperative year showed lower relative declines in lumbar spine, left hip, forearm, and total body BMD, compared to patients with lower vitamin D levels.

We could demonstrate that OAGB resulted in a large reduction in body weight by $36 \%$ in morbidly obese patients with a decrease in total body fat and an increase in lean body mass. Over 12 months, a significant decrease in BMD was seen when measured in the lumbar spine (7\%), left hip $(13 \%)$, and in total body (1\%), but not in the forearm. 
Table 1 Change in bone mineral density, parameters of bone turnover, and body composition over time

\begin{tabular}{|c|c|c|c|c|c|c|c|}
\hline & Baseline (T0) & 6 months (T6) & $P$ values + & 12 months (T12) & $P$ values + & $\begin{array}{l}\text { Difference } \\
\text { (12 months) }\end{array}$ & $\begin{array}{l}P \text { values } \\
\text { (time)+ }\end{array}$ \\
\hline \multicolumn{8}{|l|}{ Bone mineral density } \\
\hline Lumbar spine $\left(\mathrm{g} / \mathrm{cm}^{2}\right)$ & $1.09(1.07 ; 1.1)$ & $1.06(1.05 ; 1.08)$ & 0.066 & $1.01(0.99 ; 1.03)$ & $<0.001$ & $-0.08(-0.1 ;-0.05)$ & $<0.001$ \\
\hline T-score & $0.26(0.14 ; 0.38)$ & $0.07(-0.06 ; 0.2)$ & 0.113 & $-0.45(-0.63 ;-0.28)$ & $<0.001$ & $-0.71(-0.97 ;-0.45)$ & $<0.001$ \\
\hline Left hip $\left(\mathrm{g} / \mathrm{cm}^{2}\right)$ & $1.07(1.04 ; 1.11)$ & $1.03(0.99 ; 1.07)$ & 0.269 & $0.94(0.89 ; 0.99)$ & $<0.001$ & $-0.13(-0.21 ;-0.06)$ & $<0.001$ \\
\hline T-score & $0.85(0.74 ; 0.96)$ & $0.3(0.18 ; 0.41)$ & $<0.001$ & $-0.23(-0.38 ;-0.08)$ & $<0.001$ & $-1.08(-1.3 ;-0.86)$ & $<0.001$ \\
\hline Forearm $\left(\mathrm{g} / \mathrm{cm}^{2}\right)$ & $0.58(0.58 ; 0.59)$ & $0.58(0.58 ; 0.59)$ & 1.000 & $0.58(0.57 ; 0.58)$ & 0.366 & $-0.01(-0.01 ; 0)$ & 0.295 \\
\hline T-score & $-0.17(-0.26 ;-0.09)$ & $-0.2(-0.3 ;-0.11)$ & 1.000 & $-0.38(-0.51 ;-0.26)$ & 0.023 & $-0.21(-0.4 ;-0.02)$ & 0.023 \\
\hline Total body $\left(\mathrm{g} / \mathrm{cm}^{2}\right)$ & $1.12(1.11 ; 1.12)$ & $1.12(1.11 ; 1.12)$ & 1.000 & $1.1(1.09 ; 1.11)$ & 0.048 & $-0.01(-0.03 ;-0.01)$ & 0.029 \\
\hline T-score & $0.02(-0.06 ; 0.1)$ & $0.03(-0.06 ; 0.11)$ & 1.000 & $-0.15(-0.26 ;-0.04)$ & 0.030 & $-0.16(-0.32 ;-0.01)$ & 0.017 \\
\hline \multicolumn{8}{|l|}{ Bone turnover } \\
\hline Albumin (g/dl) & $45.3(44.5 ; 46.2)$ & $41.8(40.9 ; 42.8)$ & $<0.001$ & $42(41 ; 43.1)$ & $<0.001$ & $-3.3(-5 ;-1.6)$ & $<0.001$ \\
\hline CTX (ng/ml) & $0.1(0.3 ; 0.3)$ & $0.9(0.8 ; 0.9)$ & $<0.001$ & $0.9(0.9 ; 1)$ & $<0.001$ & $0.6(0.6 ; 0.7)$ & $<0.001$ \\
\hline Osteocalcin (ng/ml) & $17.1(15 ; 19.2)$ & $36.2(34 ; 38.5)$ & $<0.001$ & $41.1(38.7 ; 43.5)$ & $<0.001$ & $24(20.5 ; 27.5)$ & $<0.001$ \\
\hline $\begin{array}{l}\text { Bone-specific } \\
\text { alkaline } \\
\text { phosphatase } \\
\text { (ng/ml) }\end{array}$ & $13.4(12 ; 14.8)$ & $15.7(14.1 ; 17.2)$ & 0.050 & $18.5(16.9 ; 20.1)$ & $<0.001$ & $5.1(2.7 ; 7.5)$ & $<0.001$ \\
\hline P1NP (mg/l) & $47.2(41.5 ; 52.9)$ & $82.4(76.2 ; 88.6)$ & $<0.001$ & $90.4(83.8 ; 97.1)$ & $<0.001$ & $43.2(33.5 ; 53)$ & $<0.001$ \\
\hline iPTH (pg/ml) & $48.6(45.3 ; 51.8)$ & $51.1(47.6 ; 54.5)$ & 0.861 & $46.9(43.2 ; 50.7)$ & 0.510 & $-1.6(-7.6 ; 4.4)$ & 0.256 \\
\hline $25(\mathrm{OH}) \mathrm{D}(\mathrm{mmol} / \mathrm{l})$ & $39.6(34.6 ; 44.5)$ & $62.6(57.3 ; 67.8)$ & $<0.001$ & $63.2(57.5 ; 68.9)$ & $<0.001$ & $23.6(15.1 ; 32.1)$ & $<0.001$ \\
\hline $1,25(\mathrm{OH})_{2} \mathrm{D}(\mathrm{pg} / \mathrm{ml})$ & $47.1(43.5 ; 50.7)$ & $63.2(59.4 ; 67)$ & $<0.001$ & $66.3(62.2 ; 70.4)$ & $<0.001$ & $19.2(13.2 ; 25.2)$ & $<0.001$ \\
\hline $\mathrm{Ca}(\mathrm{mmol} / \mathrm{l})$ & $2.33(2.3 ; 2.35)$ & $2.33(2.3 ; 2.35)$ & 1.000 & $2.29(2.26 ; 2.31)$ & 0.102 & $-0.04(-0.08 ; 0.01)$ & 0.051 \\
\hline Corr. $\mathrm{Ca}(\mathrm{mmol} / \mathrm{l})$ & $2.17(2.15 ; 2.19)$ & $2.21(2.19 ; 2.23)$ & 0.027 & $2.17(2.14 ; 2.19)$ & 0.742 & $-0.01(-0.04 ; 0.03)$ & 0.008 \\
\hline Phosphate (mmol/l) & $1.05(1.01 ; 1.08)$ & $1.14(1.1 ; 1.18)$ & 0.001 & $1.24(1.2 ; 1.28)$ & $<0.001$ & $0.2(0.13 ; 0.26)$ & $<0.001$ \\
\hline \multicolumn{8}{|l|}{ Body composition } \\
\hline BMI (kg/m2) & $43.8(43.2 ; 44.5)$ & $31.9(31.2 ; 32.6)$ & $<0.001$ & $28.3(27.5 ; 29)$ & $<0.001$ & $-15.6(-16.7 ;-14.4)$ & $<0.001$ \\
\hline Weight loss (\%) & - & $26.9(25.5 ; 28.4)$ & $<0.001$ & $35.6(33.9 ; 37.3)$ & $<0.001$ & $-35.7(-33.3 ;-38.1)$ & $<0.001$ \\
\hline $\mathrm{WC}(\mathrm{cm})$ & $127.9(125.9 ; 129.9)$ & $102.1(100 ; 104.3)$ & $<0.001$ & $95.4(93 ; 97.8)$ & $<0.001$ & $-32.5(-36.1 ;-29)$ & $<0.001$ \\
\hline $\mathrm{TBF}(\%)$ & $39.4(37.3 ; 41.5)$ & $35.6(33.8 ; 37.5)$ & $<0.001$ & $32.4(29.9 ; 34.5)$ & $<0.001$ & $-7.1(-10.9 ;-3.3)$ & $<0.001$ \\
\hline $\operatorname{LBM}(\%)$ & $55.2(54.3 ; 56.1)$ & $63.8(68.4 ; 64.8)$ & $<0.001$ & $69.7(68.4 ; 70.9)$ & $<0.001$ & $14.5(12.7 ; 16.3)$ & $<0.001$ \\
\hline $\operatorname{ASM}(\%)$ & $25.0(24.5 ; 25.5)$ & $28.5(28.0 ; 29.0)$ & $<0.001$ & $31.2(30.5 ; 31.9)$ & $<0.001$ & $6.3(5.3 ; 7.2)$ & $<0.001$ \\
\hline
\end{tabular}

Note: Data are presented as mean (95\% confidence interval); “+” repeated measures analysis of variance and post hoc analysis with Bonferroni correction, adjusted for age, sex, and baseline values

$C T X$ serum type 1 collagen cross-linked C-telopeptide, $P 1 N P$ intact amino terminal propeptide of type 1 procollagen, $i P T H$ intact parathyroid hormone, $25(\mathrm{OH}) \mathrm{D}$ 25-hydroxy vitamin D, 1,25-(OH)2D 1,25-dihydroxy vitamin D, Corr. Ca corrected total calcium, $B M I$ body mass index, $W C$ waist circumference, $T B F$ total body fat, $T B F I$ total body fat index, $L B M$ lean body mass, $A S M$ appendicular skeletal muscle mass

At baseline: $n=50$, at 6 months: $n=43$, and at 12 months: $n=37$

Significant findings are in italics

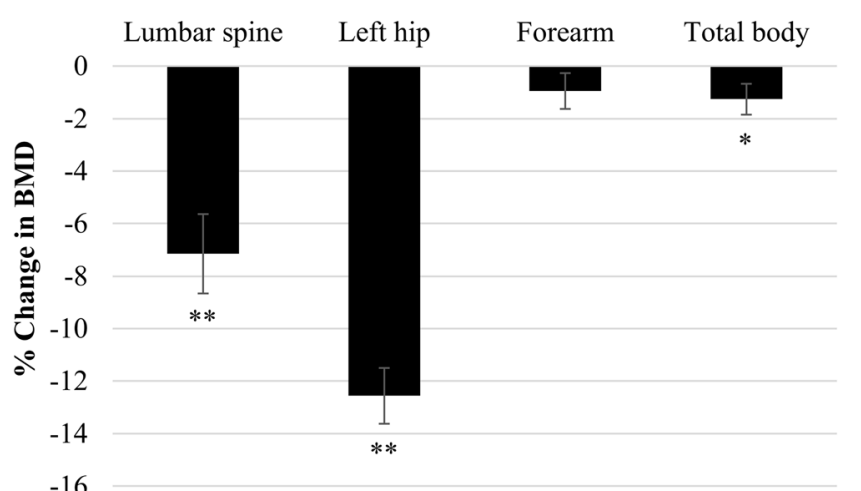

Fig. 2 Change in bone mineral density (BMD) at each measurement site 12 months after one-anastomosis gastric bypass (OAGB), as percent change in BMD from preoperative baseline. Note: BMD: bone mineral density; paired $t$ test; error bars: standard error; $* p<0.05 ; * * p<0.01$ compared with baseline
Regarding RYGB, studies on BMD are inconclusive reporting decreases between 3 and $7 \%$ in the spine [30-32] and in the hip between 8 and $11 \%$ in the first year [30-37] or no changes [35, 36] and unchanged BMD in the forearm [34-36]. BMD declines for the total body have been observed up to $3 \%[32,34]$.

Compared to RYGB studies, we found a similar decline in lumbar spine, higher in the hip, lower in total body, and likewise no changes in the forearm in patients undergoing OAGB. Moreover, 1-year weight loss ranged between 30 and $35 \%$ in RYGB studies [30-32, 35, 36], which is comparable to our study of OAGB patients who lost $36 \%$. In addition, weight loss or BMI loss was not significantly associated with the decrease in BMD. In that regard, obesity has been considered as protective against osteoporosis [18] and the decline in hip BMD in RYGB patients has been found to be associated with the surgically induced weight loss itself [35]. However, our 


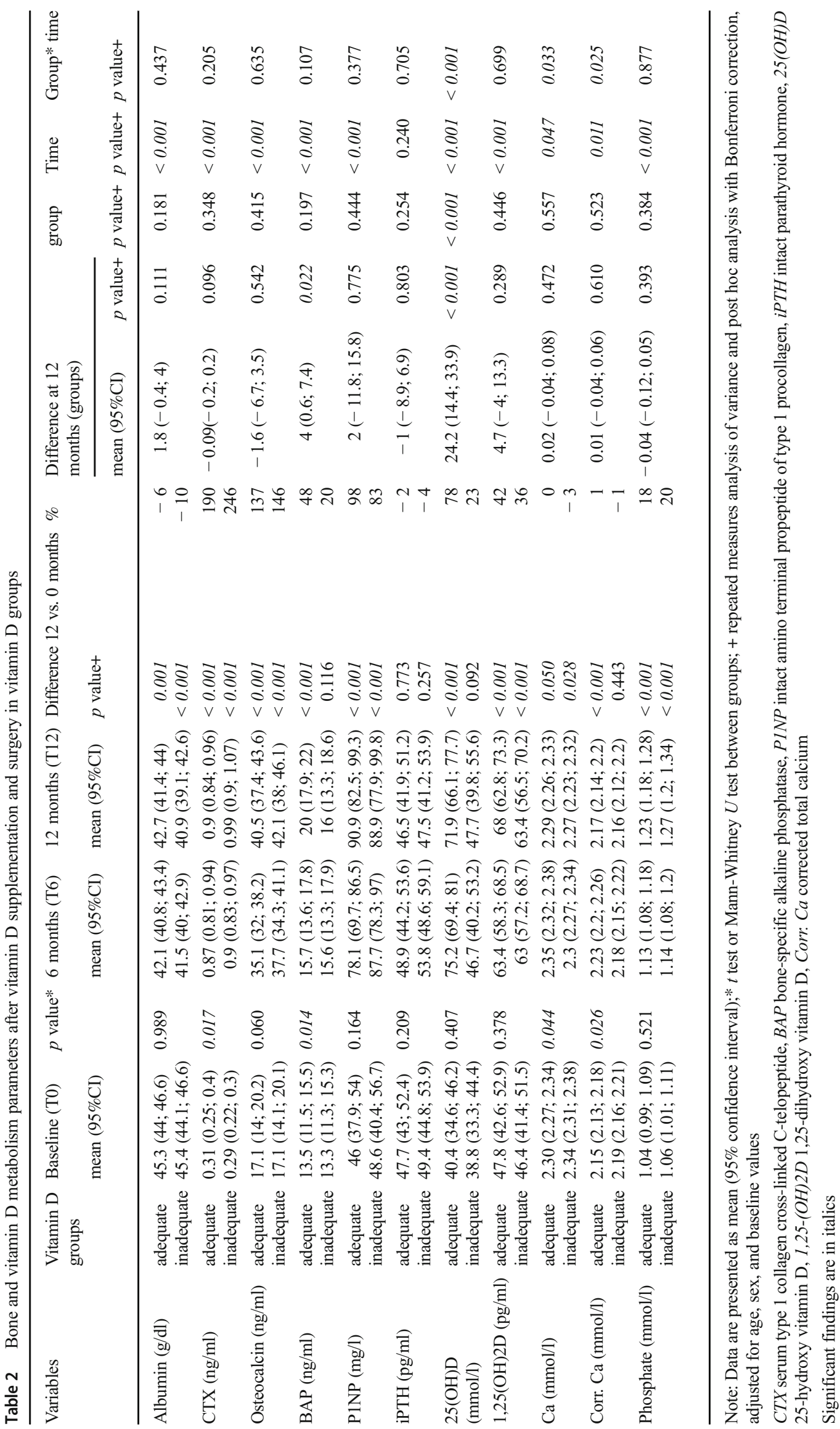


a

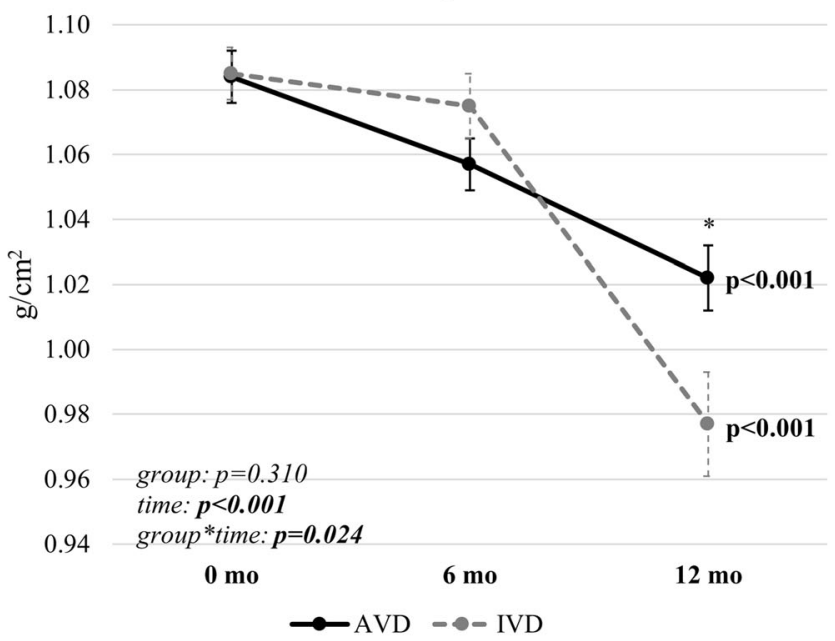

c

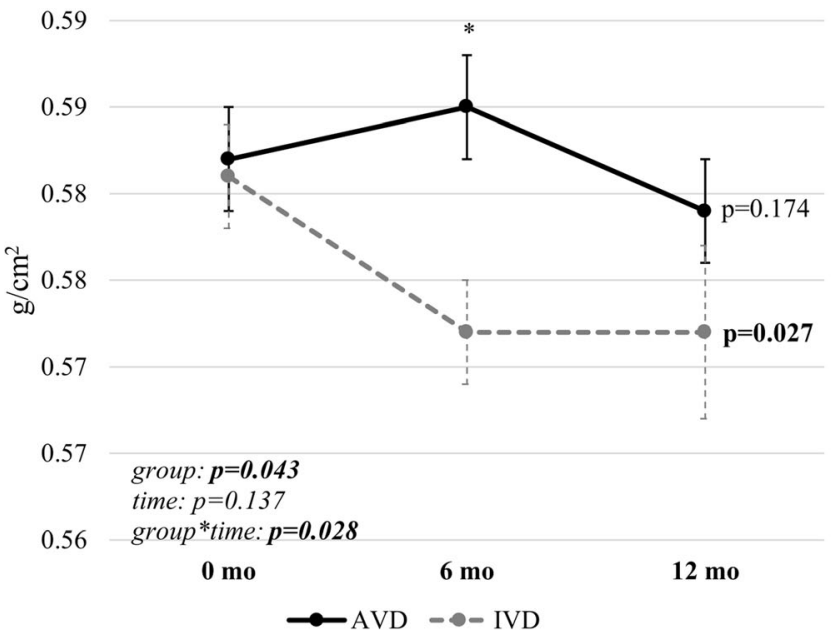

Fig. 3 Change in bone mineral density (BMD, g/ $\mathrm{cm}^{2}$ ) of lumbar spine (a), of left hip (b), of forearm (c), and of total body (d) over the time between the vitamin D groups. Note: AVD: adequate vitamin D group;

study could not find an association between bone loss and weight loss, and therefore, bone loss seems independent from surgically induced weight loss in our study population. It is noteworthy that while the percentage of the body fat mass decreased, the lean body mass as well as the appendicular skeletal muscle mass increased significantly over time. One explanation for that could be the close monitoring of the patients in the first 6 months postoperatively with individual dietary counseling every month within the study visits. In addition to that, the patients were advised to perform physical activity. Up to now, the role of vitamin D inadequacy in postoperative bone loss is not fully understood and the use of supplements has not been proven to reverse this process [20]. In that regard, we found that a lower bone loss, in left hip BMD, was associated with a higher $25(\mathrm{OH}) \mathrm{D}$ concentrations during the first postoperative year. Thus, higher vitamin b

Left Hip

1.15

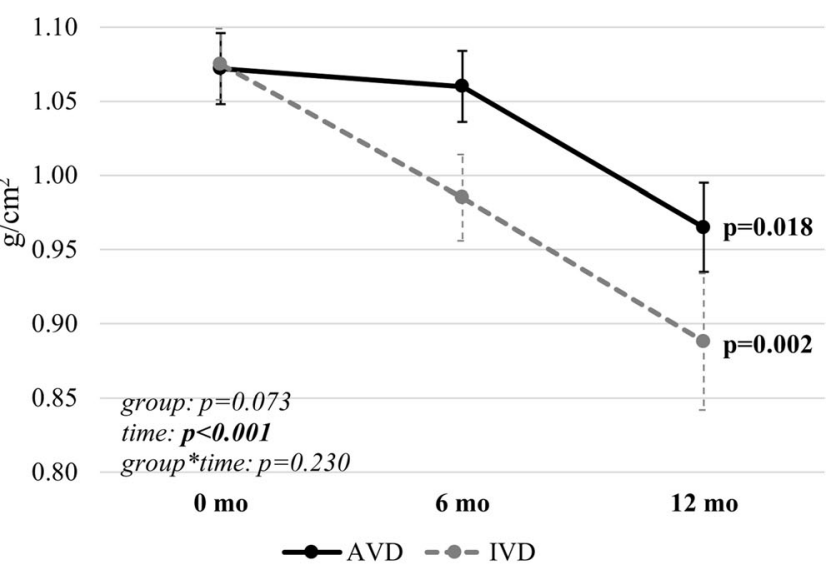

d 1.14 1.13

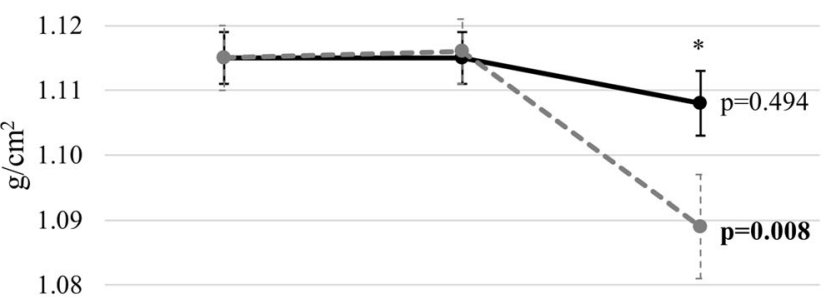

time: $\boldsymbol{p}=\mathbf{0 . 0 0 6}$

1.06

Total body
1.07 group: $p=0.253$

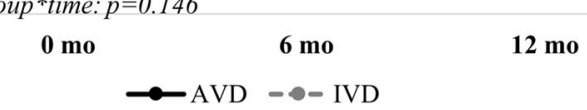

IVD: inadequate vitamin $\mathrm{D}$ group; repeated measures analysis of variance and post hoc analysis with Bonferroni correction, adjusted for age, sex, and baseline values; error bars: standard error; $* p<0.05 ; * * p<0.01$

D concentrations following high-dose vitamin D supplementation in the first postoperative year might be able to decelerate bone loss, which is in accordance with studies in elderly women [14]. A supplementation with $400 \mathrm{IU}$ vitamin $\mathrm{D}_{3}$ daily in elderly women slightly decreased iPTH levels and increased BMD [14]. In RYGB patients, the rate of bone loss is greatest in the first postoperative year and plateaus or even slightly improves in subsequent years [19]. Therefore, it is crucial to maintain high the $25(\mathrm{OH}) \mathrm{D}$ concentrations in the first postoperative year. In addition, regarding malabsorption, we found no significant differences between the vitamin $\mathrm{D}$ groups in weight loss and all study participants underwent an OAGB with the same alimentary limb length of $200 \mathrm{~cm}$.

To the best of our knowledge, only two studies measured BMD in RYGB patients in relation to different high-dose standardized vitamin D supplementation regimes. Muschitz 
a

Lumbar spine

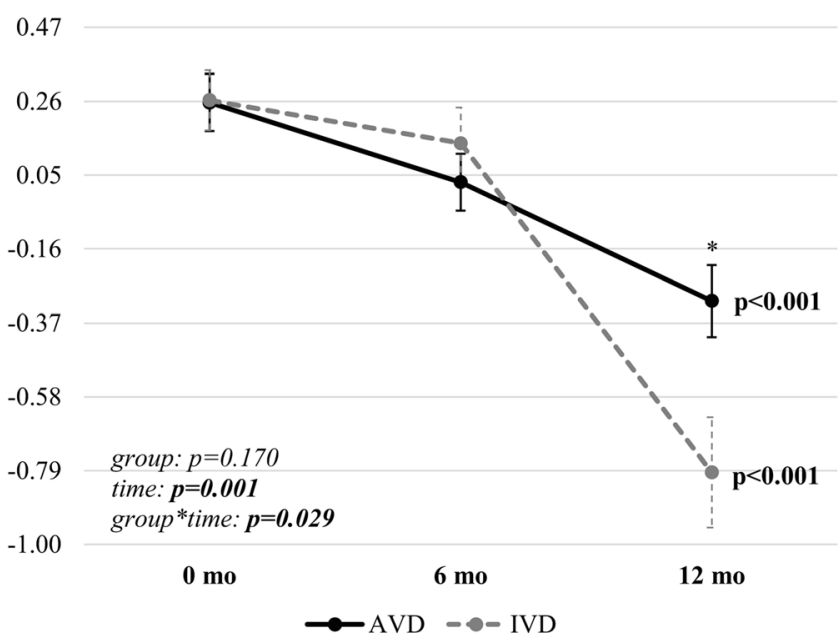

c

Forearm

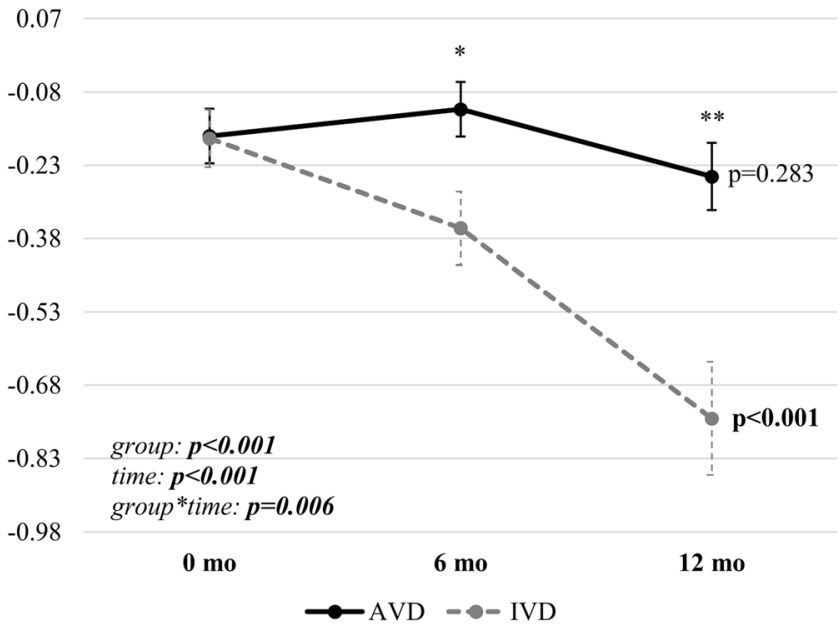

Fig. 4 Change in bone mineral density (BMD) t-score of lumbar spine (a), of left hip (b), of forearm (c), and of total body (d) over the time between the vitamin D groups. Note: AVD: adequate vitamin D group;

et al. studied the impact of vitamin D, calcium, protein supplementation, and physical exercise (Nordic walking, strength b

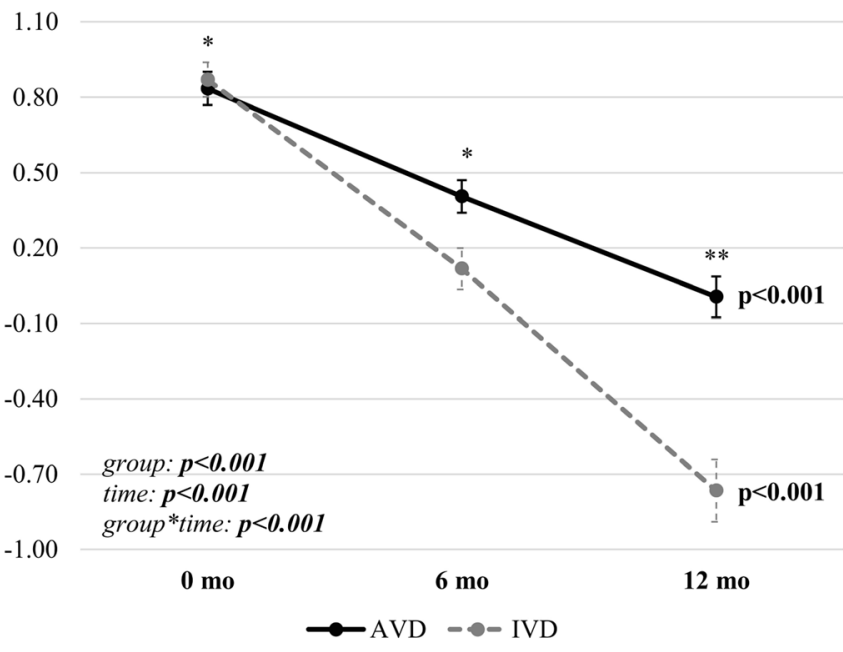

d

0.12

0.02

$-0.08$

$-0.17$

$-0.27$

$-0.36$

$-0.46$

$-0.55$

time: $\boldsymbol{p}=\mathbf{0 . 0 0 1}$

group $*$ time: $\boldsymbol{p}=\mathbf{0 . 0 2 6}$

0 mo

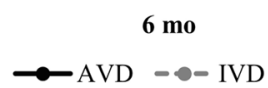

IVD: inadequate vitamin $\mathrm{D}$ group; repeated measures analysis of variance and post hoc analysis with Bonferroni correction, adjusted for age, sex, and baseline values; error bars: standard error; $* p<0.05 ; * * p<0.01$

perseverance, and equipment training) on bone metabolism after RYGB and sleeve gastrectomy. Patients in the

Table 3 Estimates of the prevalence of osteopenia (t-score below - 1) after vitamin D supplementation and surgery in vitamin D groups

\begin{tabular}{|c|c|c|c|c|c|c|c|c|c|}
\hline $\mathrm{BMD}$ region & Vitamin D groups & Baseline (T0) & $p$ value* & 6 months (T6) & $p$ value* & 12 months (T12) & $p$ value* & $p$ value time* & $p$ value group* \\
\hline Lumbar spine & $\begin{array}{l}\text { adequate } \\
\text { inadequate }\end{array}$ & $\begin{array}{r}10 \% \\
6 \%\end{array}$ & 0.563 & $\begin{array}{l}8 \% \\
5 \%\end{array}$ & 0.675 & $\begin{array}{l}62 \% \\
49 \%\end{array}$ & 0.515 & 0.001 & 0.523 \\
\hline Left hip & $\begin{array}{l}\text { adequate } \\
\text { inadequate }\end{array}$ & $\begin{array}{l}0 \% \\
0 \%\end{array}$ & 0.768 & $\begin{array}{l}4 \% \\
6 \%\end{array}$ & 0.765 & $\begin{array}{l}38 \% \\
45 \%\end{array}$ & 0.758 & $<0.001$ & 0.757 \\
\hline Forearm & $\begin{array}{l}\text { adequate } \\
\text { inadequate }\end{array}$ & $\begin{array}{l}15 \% \\
26 \%\end{array}$ & 0.343 & $\begin{array}{l}14 \% \\
25 \%\end{array}$ & 0.351 & $\begin{array}{l}17 \% \\
29 \%\end{array}$ & 0.390 & 0.921 & 0.340 \\
\hline Total body & $\begin{array}{l}\text { adequate } \\
\text { inadequate }\end{array}$ & $\begin{array}{l}19 \% \\
19 \%\end{array}$ & 0.974 & $\begin{array}{l}11 \% \\
12 \%\end{array}$ & 0.974 & $\begin{array}{l}29 \% \\
30 \%\end{array}$ & 0.974 & 0.038 & 0.974 \\
\hline
\end{tabular}

Note: Data are presented as estimates of the prevalence in $\% *$ Generalized estimating equation, adjusted for age, sex, season, and baseline values Significant findings are in italics 
intervention group received 28,000 IU cholecalciferol/week for 8 weeks before and 16,000 IU/week after bariatric surgery [38]. Patients in our study received either three oral vitamin $\mathrm{D}_{3}$ loading doses of each 100,000 IU, corresponding to an average of 70,000 IU cholecalciferol/week in the first months postoperatively (loading) or placebo, and all of them obtained a daily maintenance dose of 3420 , on average $24,000 \mathrm{IU} /$ week until 6 months after surgery with compliance testing. Thereafter, patients were instructed to continue vitamin $\mathrm{D}_{3}$ supplementation until the follow-up visit at 12 months [23]. The study by Muschitz et al. concluded that a combination of physical exercise with vitamin D, calcium, and BMI-adjusted protein supplementation decelerates the loss of areal BMD and lean body mass after 2 years of RYGB surgery [38]. However, it is not possible to dissect the isolated effects of the respective interventions, in particular of vitamin D supplementation [38]. In another randomized vitamin $\mathrm{D}_{3}$ supplementation trial, patients in the intervention group received 50,000 cholecalciferol/week in addition to $5600 \mathrm{IU} /$ week compared with a control group of only 5600 IU/week until 1 year after RYGB surgery [39]. The authors of this study demonstrated that 50,000 IU of vitamin D weekly after RYGB attenuates cortical bone loss [39].

As a result of decreased vitamin D absorption or concentration, elevated iPTH or secondary hyperparathyroidism can exert negative effects on BMD. Therefore, an adequate level of vitamin $\mathrm{D}$ ( $\geq 50 \mathrm{nmol} / \mathrm{l})$ might be required to keep the iPTH level in the normal range. In our study population, iPTH and calcium concentrations remained unchanged and no patient demonstrated high iPTH levels after one postoperative year. This is in contrast to the findings of our previously published prospective cohort study in bariatric patients receiving a lower and non-standardized vitamin D supplementation $(95 \% \mathrm{CI}$ 200-3000 IU/day according to the available guidelines at that time) with a prevalence of high iPTH levels of $23 \%$ [8]. Thus, adequate vitamin $\mathrm{D}$ concentrations are needed to prevent an increase in iPTH levels [17].

Lower bone loss in total body was associated with lower bone turnover markers such as CTX, osteocalcin, and P1NP values over the study duration of 1 year. These bone turnover markers, CTX, osteocalcin, bone-specific alkaline phosphatase, and P1NP, increased significantly, as well as 25(OH)D, $1,25(\mathrm{OH})_{2} \mathrm{D}$, and phosphate, after 12 months. On the contrary, albumin, and albumin corrected calcium decreased while iPTH concentrations remained unchanged compared to baseline. Similarly to our previously published cohort study [8], we observed an approximately same high prevalence of high bone formation markers (CTX and osteocalcin) after 12 months.

Several limitations have to be mentioned. First, the sample size is rather small, although it was based on the sample size calculation taking into account differences of serum $25(\mathrm{OH}) \mathrm{D}$ concentrations at 6 months between the intervention and control group. Indeed, we were able to demonstrate significant differences, which validate the sample size calculation. Our study included a high percentage of women $(80 \%)$, which, however, is very common in bariatric patients. Due to the body weight limitation of the DXA procedure, body weight of the patients was below $140 \mathrm{~kg}$ before surgery, which, however, is representative for $82 \%$ of our bariatric patient cohort undergoing OAGB [8]. Another limitation of this study is the lack of physical activity measurements, as physical exercise might have an impact on BMD. Furthermore, this study was not designed to evaluate any potential clinical risks or benefits such as fracture outcome of the investigated population.

Nevertheless, the significant and relevant novel findings, the design of the study provides detailed pre- and postoperative data of patients undergoing OAGB, which, as a rather new bariatric procedure, has not been evaluated in that regard.

In conclusion, our study reports in patients undergoing the relatively new OAGB compared to those with RYGB a similar decline of BMD in lumbar spine, higher in the hip, lower in total body with no changes in the forearm. As can be expected, bone turnover markers increased significantly in the first postoperative year but all patients demonstrated normal iPTH concentrations, most probably due to higher vitamin D concentrations. Furthermore, bone loss seems independent from surgically induced weight loss in our patients, and lower bone loss was associated with higher 25(OH)D concentrations. Accordingly, the relative declines in lumbar spine, left hip, forearm, and total body BMD were lower in those patients with higher $25(\mathrm{OH}) \mathrm{D}$ concentrations ( $\geq 50 \mathrm{nmol} / \mathrm{l}$ ) after 6 and 12 months compared to patients with lower concentrations. Thus, higher vitamin D concentrations during the first postoperative year following adequate vitamin D supplementation might be able to decelerate bone loss. Although higher vitamin D levels are challenging to reach during weight loss, patients undergoing OAGB need to keep the $25(\mathrm{OH}) \mathrm{D}$ concentrations above $50 \mathrm{nmol} / \mathrm{l}$, at least over the first postoperative year, to decelerate bone loss.

Acknowledgements We would like to thank Carmen Klammer, Miranda Adelfang, Michaela Faustmann, and Hacer Geyik for their support. We would also like to thank Alexandra Kaider for the statistical input and, last but not least, the bariatric patients who participated in this study.

Authors' Contributions The project idea came from ML, RK, RM, and BL. ML, RK, RM, KS, FK, FH, FL, GP, and BL designed the research. ML, RK, FK, and MK conducted the research. ML, EW, and IG analyzed the data, performed statistical analyses, and drafted the manuscript with appreciable input from RK, KS, FK, RM, MK, FL, GP, and BL. ML and BL had prime responsibility for the final manuscript content. All authors read and approved the final manuscript.

Funding Information Open access funding provided by Medical University of Vienna. The work of this paper was partly supported by the Austrian Biobanking and BioMolecular resources Research Infrastructure 
(BBMRI.at) funded by the Austrian Federal Ministry of Science, Research and Economy (BMWFW GZ 10.470/0016-II/3/2013), by the non-profit organization "Special Institute for Preventive Cardiology And NutritionSIPCAN" (Salzburg) for personnel costs and Fresenius Kabi supplied, cost-free, the Oleovit and placebo oil but all had no role in the design and conduct of the study, the collection, analysis, and interpretation of data, in the preparation of the manuscript, or in the review or approval of the manuscript.

\section{Compliance with Ethical Standards}

This study was approved by the local Ethics Committee of the Medical University of Vienna (No. 1899/2013), by the Austrian Competent Authority (No. LCM-718280-0001), registered at clinicaltrials.gov (Identifier: NCT02092376) and EudraCT (Identifier: 2013-003546-16), complies with the Declaration of Helsinki [22], and conducted from April 2014 to June 2016 at the Medical University of Vienna (Austria). The study participants were scheduled for OAGB surgery and all of them gave written, informed consent preoperatively.

Conflict of Interest All authors have no commercial associations that might be a conflict of interest in relation to this article.

Open Access This article is distributed under the terms of the Creative Commons Attribution 4.0 International License (http:// creativecommons.org/licenses/by/4.0/), which permits unrestricted use, distribution, and reproduction in any medium, provided you give appropriate credit to the original author(s) and the source, provide a link to the Creative Commons license, and indicate if changes were made.

\section{References}

1. Buchwald H, Avidor Y, Braunwald E, et al. Bariatric surgery: a systematic review and meta-analysis. JAMA. 2004;292(14):172437.

2. Sjostrom L. Review of the key results from the Swedish obese subjects (SOS) trial - a prospective controlled intervention study of bariatric surgery. J Intern Med. 2013;273(3):219-34.

3. Sasaki A, Nitta H, Otsuka K, et al. Bariatric surgery and nonalcoholic fatty liver disease: current and potential future treatments. Front Endocrinol (Lausanne). 2014;5:164.

4. Sjostrom L, Narbro K, Sjostrom CD, et al. Effects of bariatric surgery on mortality in Swedish obese subjects. N Engl J Med. 2007;357(8):741-52.

5. Bacci V, Silecchia G. Vitamin D status and supplementation in morbid obesity before and after bariatric surgery. Expert Rev Gastroenterol Hepatol. 2010;4(6):781-94.

6. Ducloux R, Nobecourt E, Chevallier JM, et al. Vitamin D deficiency before bariatric surgery: should supplement intake be routinely prescribed? Obes Surg. 2011;21(5):556-60.

7. Fish E, Beverstein G, Olson D, et al. Vitamin D status of morbidly obese bariatric surgery patients. J Surg Res. 2010;164(2):198-202.

8. Luger M, Kruschitz R, Langer F, et al. Effects of omega-loop gastric bypass on vitamin $\mathrm{D}$ and bone metabolism in morbidly obese bariatric patients. Obes Surg. 2015;25(6):1056-62.

9. Hage MP, El-Hajj Fuleihan G. Bone and mineral metabolism in patients undergoing roux-en-Y gastric bypass. Osteoporos Int. $2014 \mathrm{Feb} ; 25(2): 423-39$.
10. Cranney A, Horsley T, O'Donnell S, Weiler H, Puil L, Ooi D, et al. Effectiveness and safety of vitamin D in relation to bone health. Evid Rep Technol Assess (Full Rep) 2007 (158):1-235.

11. Stein EM, Silverberg SJ. Bone loss after bariatric surgery: causes, consequences, and management. Lancet Diabetes Endocrinol. 2014 Feb;2(2):165-74.

12. Disse E, Pasquer A, Espalieu P, et al. Greater weight loss with the omega loop bypass compared to the roux-en- $Y$ gastric bypass: a comparative study. Obes Surg. 2014;24(6):841-6.

13. Mahawar KK, Sharples AJ. Contribution of Malabsorption to weight loss after roux-en-Y gastric bypass: a systematic review. Obes Surg. 2017;27(8):2194-206.

14. Ooms ME, Roos JC, Bezemer PD, et al. Prevention of bone loss by vitamin D supplementation in elderly women: a randomized double-blind trial. J Clin Endocrinol Metab. 1995;80(4):1052-8.

15. Bischoff-Ferrari HA, Dawson-Hughes B, Willett WC, et al. Effect of vitamin D on falls: a meta-analysis. JAMA. 2004;291(16):1999 2006.

16. Ricci TA, Chowdhury HA, Heymsfield SB, et al. Calcium supplementation suppresses bone turnover during weight reduction in postmenopausal women. J Bone Miner Res. 1998;13(6):1045-50.

17. Goldner WS, Stoner JA, Lyden E, et al. Finding the optimal dose of vitamin $\mathrm{D}$ following roux-en-Y gastric bypass: a prospective, randomized pilot clinical trial. Obes Surg. 2009;19(2):173-9.

18. Krolner B, Ranlov PJ, Clemmesen T, et al. Bone loss after gastroplasty for morbid obesity: side-effect or adaptive response to weight reduction? Lancet. 1982;1(8278):956-7.

19. Johnson JM, Maher JW, Samuel I, et al. Effects of gastric bypass procedures on bone mineral density, calcium, parathyroid hormone, and vitamin D. J Gastrointest Surg. 2005;9(8):1106-10.

20. Zalesin KC, Miller WM, Nori Janosz KE, et al. Controversies in vitamin D: deficiency and supplementation after roux-en-Y gastric bypass surgery. Therapy. 2007;4(5):561-74.

21. Luger M, Kruschitz R, Kienbacher C, et al. Vitamin D3 loading is superior to conventional supplementation after weight loss surgery in vitamin D-deficient morbidly obese patients: a double-blind randomized placebo-controlled trial. Obes Surg. 2017;27(5):1196207.

22. Dale $\mathrm{O}$, Salo $\mathrm{M}$. The Helsinki declaration, research guidelines and regulations: present and future editorial aspects. Acta Anaesthesiol Scand. 1996;40(7):771-2.

23. Luger M, Kruschitz R, Marculescu R, et al. The link between obesity and vitamin $\mathrm{D}$ in bariatric patients with omega-loop gastric bypass surgery - a vitamin D supplementation trial to compare the efficacy of postoperative cholecalciferol loading (LOAD): study protocol for a randomized controlled trial. Trials. 2015;16(1):328.

24. Rutledge R. The mini-gastric bypass: experience with the first 1 , 274 cases. Obes Surg. 2001;11(3):276-80.

25. Schulz KF, Altman DG, Moher D, et al. CONSORT 2010 statement: updated guidelines for reporting parallel group randomised trials. Trials. 2010;11:32.

26. Genant HK, Cooper C, Poor G, et al. Interim report and recommendations of the World Health Organization task-force for osteoporosis. Osteoporos Int. 1999;10(4):259-64.

27. International Society for the Advancement of Kinanthropometry (ISAK). International Standards for Anthropometric Assessment. 2001; Available from: http://www.ceap.br/material/ MAT17032011184632.pdf.

28. Jain A, Bhayana S, Vlasschaert $\mathrm{M}$, et al. A formula to predict corrected calcium in haemodialysis patients. Nephrol Dial Transplant. 2008 Sep;23(9):2884-8.

29. Zhang S, Paul J, Nantha-Aree M, et al. Empirical comparison of four baseline covariate adjustment methods in analysis of continuous outcomes in randomized controlled trials. Clin Epidemiol. 2014;6:227-35. 
30. Pereira FA, de Castro JA, dos Santos JE, et al. Impact of marked weight loss induced by bariatric surgery on bone mineral density and remodeling. Braz J Med Biol Res. 2007;40(4):509-17.

31. Vilarrasa N, Gomez JM, Elio I, et al. Evaluation of bone disease in morbidly obese women after gastric bypass and risk factors implicated in bone loss. Obes Surg. 2009;19(7):860-6.

32. Carrasco F, Ruz M, Rojas P, et al. Changes in bone mineral density, body composition and adiponectin levels in morbidly obese patients after bariatric surgery. Obes Surg. 2009 Jan;19(1):41-6.

33. Vilarrasa N, San Jose P, Garcia I, et al. Evaluation of bone mineral density loss in morbidly obese women after gastric bypass: 3-year follow-up. Obes Surg. 2011;21(4):465-72.

34. Coates PS, Fernstrom JD, Fernstrom MH, et al. Gastric bypass surgery for morbid obesity leads to an increase in bone turnover and a decrease in bone mass. J Clin Endocrinol Metab. 2004;89(3): 1061-5.
35. Fleischer J, Stein EM, Bessler M, et al. The decline in hip bone density after gastric bypass surgery is associated with extent of weight loss. J Clin Endocrinol Metab. 2008;93(10):3735-40.

36. Stein EM, Carrelli A, Young P, et al. Bariatric surgery results in cortical bone loss. J Clin Endocrinol Metab. 2013;98(2):541-9.

37. Nielson CM, Srikanth P, Orwoll ES. Obesity and fracture in men and women: an epidemiologic perspective. J Bone Miner Res. 2012;27(1):1-10.

38. Muschitz C, Kocijan R, Haschka J, et al. The impact of vitamin D, calcium, protein supplementation, and physical exercise on bone metabolism after bariatric surgery: the BABS study. J Bone Miner Res. 2016;31(3):672-82.

39. Carlin AM, Rao DS, Yager KM, et al. Treatment of vitamin D depletion after roux-en-Y gastric bypass: a randomized prospective clinical trial. Surg Obes Relat Dis. 2009;5(4):444-9.

\section{Affiliations}

\section{Maria Luger ${ }^{1,2,3} \cdot$ Renate Kruschitz ${ }^{1,4} \cdot$ Eva Winzer $^{2,3}$ (1) Karin Schindler $^{1} \cdot$ Igor Grabovac $^{3} \cdot$ Franz Kainberger $^{5}$. Michael Krebs ${ }^{1}$ • Friedrich Hoppichler ${ }^{2,6} \cdot$ Felix Langer $^{7}$ • Gerhard Prager ${ }^{7} \cdot$ Rodrig Marculescu $^{8}$ • Bernhard Ludvik ${ }^{1,9}$}

1 Division of Endocrinology and Metabolism, Department of Internal Medicine III, Medical University of Vienna, Währinger Gürtel 1820, 1090 Vienna, Austria

2 Special Institute for Preventive Cardiology And Nutrition-SIPCAN, Rabenfleckweg 8, Elsbethen, 5061 Salzburg, Austria

3 Department of Social and Preventive Medicine, Centre for Public Health, Medical University of Vienna, Kinderspitalgasse 15/1, 1090 Vienna, Austria

4 Division of Internal Medicine, General public hospital of the Order of Saint Elisabeth, Völkermarkter Straße 15-19, 9020 Klagenfurt, Austria

5 Division of Neuroradiology and Musculoskeletal Radiology, Department of Biomedical Imaging and Image-guided Therapy,
Medical University of Vienna, Währinger Gürtel 18-20, 1090 Vienna, Austria

6 Division of Internal Medicine, Krankenhaus der Barmherzigen Brüde Salzburgr, Kajetanerplatz 1, 5010 Salzburg, Austria

7 Division of General Surgery, Department of Surgery, Medical University of Vienna, Währinger Gürtel 18-20, 1090 Vienna, Austria

8 Clinical Institute for Medical and Chemical Laboratory Diagnostics, Department of Laboratory Medicine, Medical University of Vienna, Währinger Gürtel 18-20, 1090 Vienna, Austria

9 Department of Medicine 1 and Karl Landsteiner Institute for Obesity and Metabolic Diseases, Rudolfstiftung Hospital, Juchgasse 25, 1030 Vienna, Austria 